\title{
How Might X-Ray Scanned Potatoes Improve Cancer Treatment?
}

\author{
$\underline{\text { José-Alvim Berkenbrock }}^{1,2, *}$, Rafaela Grecco Machado ${ }^{3}$ and Sven Achenbach ${ }^{1,2}$
}

1. Synchrotron Laboratory of Micro and Nano Devices, Canadian Light Source, Saskatoon, Canada.

2. Department of Electrical and Computer Engineering/University of Saskatchewan, Saskatoon, Canada.

3. Department of Anatomy and Cell Biology/University of Saskatchewan, Saskatoon, Canada.

* Corresponding author, j.alvim@usask.ca

Electrochemotherapy (ECT) is an antitumor treatment that comprises the action of chemotherapy drugs enhanced by electric field pulses delivered locally over a target tumour [1]. The electric pulses promote the reduction of the cell membrane selectivity through a phenomenon called electropermeabilization (or electroporation, EP) [1-3]. Neither the EP protocol nor the ECT treatment provoke any observable effect in the treated tissue right after the application is done. In fact, animal tissues show no other indication of treatment success than tumour shrinkage and disappearance, called Complete Response (CR). Notwithstanding these outcomes are an obvious (and desired) indication of treatment's effectiveness, no tangible feedback is offered to clinicians earlier than 4-10 weeks [4]. In this sense, the present work aims to discuss a tentative approach to expedite treatment effectiveness feedback based on chemical species variations due to EP of tissue cells.

Despite the fact that electroporation is the current theory to explain the EP phenomenon, and the large majority of theoretical and experimental studies follow this trend, the mechanisms of pore formation still need to be unveiled. Electroporation theory for EP considers the formation of pores around cell poles. The pores' small sizes (few nanometres), and short duration (few microseconds) have imposed great challenges to their observation [2-3-5]. Single-cell EP has been observed indirectly by insertion of fluorescent dye elements (e.g. thallium ions, propidium iodide, AF-546 siRNA) [3-6] or quantum dots [7]. However, these techniques are not indicated to live subjects during ongoing treatments, being restricted to in vitro experiments. To avoid the use of dyes, an option is to use tissues where the EP do leave observable signs, like in potatoes, which electropermeabilized area become darker after 6-12 hours [8-9]. This "in vitro simulation" has the differences in tissue's constitution (i.e. cell wall) as a disadvantage, but the 3Rs concept for animal testing compliance (Reduction of number of animals; Refinement of procedures to reduce distress; and Replacement of animal with non-animal techniques), and the faster feedback as the main advantages [9-10]. Potato has been used as a model for EP studies since 2004 [8] (e.g. for testing new arrangements of electrodes [11], modelling [10], or in MRI [9]).

The lipids reorganization and hydrophilic opening that happen during pore formation in the membrane allow the unusual migration of chemical species, therefore, the track of such migration should possibly be observed with x-ray microscopy. The natural difference between intra- and extracellular concentration of chemical species is strongly affected by the presence of pores in the lipid membrane. The ion of calcium $\left(\mathrm{Ca}^{+2}\right)$ has been suggested as a marker of EP [12]. Recently, x-ray fluorescence has allowed the micrograph of $\mathrm{Ca}^{+2}$ and many other elements (like phosphor, sulphur, potassium) in biological tissues [13]. Resolution-wise, the order of millimetres would be enough to allow the observation of macroscopic signs of treatment [9], but the submicrometers scale is also aimed to achieve closer observation of cells.

By the day of the Congress, beam lines options and the required capabilities will be discussed to 
determine the suitability. The device for delivering electric pulses and the overall scheme will be presented. Predicted outcomes include validation of proposed methodological approach and future application of this technique with ECT treatments. The validation relies on performing initial tests with potato for approving $\mathrm{Ca}^{+2}$ as EP marker or screening for other candidates. After that, animal tissues must be assessed too. Be able to 'see' that the tumour cells were not permeabilized at a first application of ECT would allow clinicians to do another ECT session quickly, reducing the chance of recurrence due to non-treated tumour regions.

In conclusion, this is a pilot work in the initial stages. However, the predicted impact of implementing xrays as a instantaneous feedback for clinicians besides ECT treatments is a motivating force. Moreover, the potential for improvements in the current understanding of EP is encouraging. Participate on XRM2018 will certainly aggregate immeasurable opportunities to this work.

\section{References:}

[1] KF Chu and DE Dupuy, Nat. Rev. Cancer 14 (2014), p. 199-208.

[2] JC Weaver in "Electrical Trauma", eds. RC Lee, EG Cravalho, and JF Burke, (Cambridge University Press, Cambridge) p. 301-326.

[3] A Paganin-Gioanni et al, Proc. Nat. Acad. Sci. U.S.A. 108 (2011), p. 10443-10447.

[4] LM Mir et al, Eur. J. Cancer Supplements 4 (2006), p. 14-25.

[5] JT Sengel and MI Wallace, Proc. Nat. Acad. Sci. U.S.A. 113 (2016) ,5281-5286.

[6] AG Pakhomov et al, Biochim. Biophys. Acta, Biomembr. 1848 (2015), p. 958-966.

[7] Y Sun et al, MRS Proceedings 873 (2005) p. 1-6.

[8] LF Cima and LM Mir, Appl. Phys. Lett. 85 (2004), p. 4520.

[9] M Hjouj and B Rubinsky, J. Membr. Biol. 236 (2010), p. 137-146.

[10] JA Berkenbrock et al, J Med Biol. Eng. (in press).

[11] M Castiello et al, IEEE Trans. Dielectr. Electr. Insul. 21 (2014), p. 1424-1432.

[12] H Hanna et al, Sci. Rep. 7 (2017), p. 13079.

[13] S Vogt et al, Microsc. Microanal. 20 (2014) p. 1306-1307.

[14] The authors acknowledge funding from their Departments.

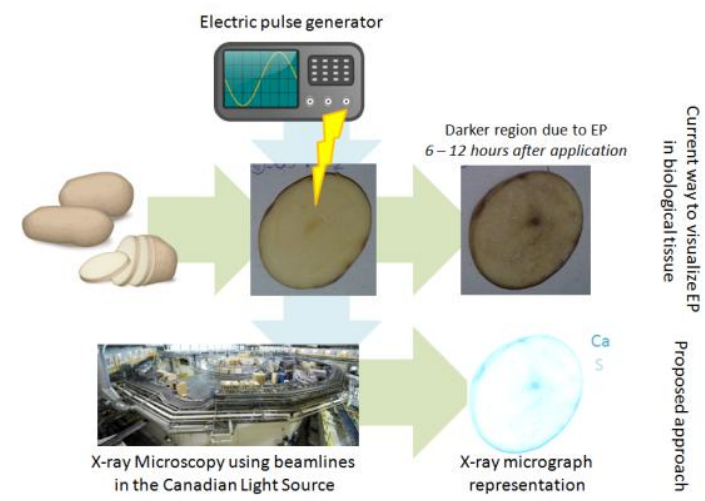

Figure. 1. Scheme of the proposed methodology. Electropermeabilized potato slices (centre) become darker after 6-12 hours (top right). The proposed approach (bottom) considers experiments in x-ray beamlines to obtain micrographs of $\mathrm{Ca}^{+2}$ in the tissue (right bottom). 